FSU TPI 02/03

\title{
Energy Density of Vortices in the Schrödinger Picture
}

\author{
J.D. Länge*, M. Engelhardt ${ }^{\dagger}$ and H. Reinhardt ${ }^{\dagger}$ \\ * Theoretisch-Physikalisches Institut, Friedrich-Schiller-Universität Jena, \\ Fröbelstieg 1, 07743 Jena, Germany \\ $\dagger$ Institut für Theoretische Physik, Eberhard-Karls-Universität Tübingen, \\ Auf der Morgenstelle 14, 72076 Tübingen, Germany
}

\begin{abstract}
The one-loop energy density of an infinitely thin static magnetic vortex in $S U(2)$ Yang-Mills theory is evaluated using the Schrödinger picture. Both the gluonic fluctuations as well as the quarks in the vortex background are included. The energy density of the magnetic vortex is discussed as a function of the magnetic flux. The center vortices correspond to local minima in the effective potential. These minima are degenerated with the perturbative vacuum if the fermions are ignored. Inclusion of fermions lifts this degeneracy, raising the vortex energy above the energy of the perturbative vacuum.

PACS numbers: $02.30 . S a, 02.30 . \mathrm{Tb}, 03.70 .+\mathrm{k}, 11.15 .-\mathrm{q}$
\end{abstract}

Keywords: center vortices, self-adjoint extensions, one-loop energy

\footnotetext{
* J.D.Laenge@tpi.uni-jena.de

$\dagger$ engelm@tphys.physik.uni-tuebingen.de, hugo.reinhardt@uni-tuebingen.de
} 


\section{INTRODUCTION}

Recently, there has been a revival of the vortex picture of confinement 1]. Lattice calculations performed in the so-called maximal center gauge with subsequent center projection 2] (which provides a simple method for identifying the center vortices in Yang-Mills configurations) have given substantial support to the idea that center vortices are the relevant infrared degrees of freedom responsible for confinement. The deconfinement phase transition in the vortex picture takes the guise of a percolation transition [3, 4]. Furthermore, the topological charge of the vortices can be understood in terms of their self-intersection number [5, 6, 7, 8, 9] and the spontaneous breaking of chiral symmetry can be generated through vortex effects [10, 11].

On the other hand, while a successful phenomenological model of vortex dynamics has been developed [4, 6, 11], investigations of the dynamical properties of Yang-Mills vortices have only recently been launched, cf. [12, 13] for thick center vortices and [14] for thin vortices of arbitrary flux. In the present paper, we expand on these investigations, which only considered gluonic fluctuations to one-loop order, by evaluating the one-loop energy density of an infinitely thin static magnetic vortex including quark fluctuations. For this purpose we use the Schrödinger picture. We solve the Schrödinger equation for gluonic fluctuations and fermions moving in the vortex background field. From the obtained eigenenergies we calculate the static energy density of the vortex as a function of its magnetic flux. We will show that the inclusion of fermions substantially changes the energy density of the vortex. In particular we will find that the fermions raise the center vortex effective energy compared with the perturbative vacuum.

The organization of the paper is as follows: In the next section we study the Schrödinger equation for the gluonic fluctuations around the vortex background field. In subsection ЩA we study the Hamiltonian of the gluonic fluctuations in the vortex background and discuss its self-adjoint extensions in subsection $\amalg B$. Subsection $\llbracket \mathrm{C}$ is devoted to the incorporation of Gauss' law and to the selection of the appropriate self-adjoint extension of the Hamiltonian of the gluonic fluctuations. In subsection $11 \mathrm{D}$ the gluonic contribution to the energy density of the vortex is presented.

In section III we study fermions in the vortex background field and calculate their contribution to the energy density. The physical interpretation of our results and some concluding 
remarks are given in section IV]

\section{GLUONIC FLUCTUATIONS AROUND THE VORTEX BACKGROUND FIELD}

\section{A. The Hamiltonian of the gluonic fluctuations}

Our aim is to evaluate the one-loop corrections to the energy density of a static SU(2) vortex background field. We consider an infinitely thin vortex of magnetic flux $\Phi$ on the 3-axis described by a gauge potential

$$
\overline{\boldsymbol{A}}(\rho, \varphi)=\frac{\sigma^{3}}{2} \frac{\Phi}{g \rho} \hat{\boldsymbol{e}}_{\varphi},
$$

where the polar coordinates $(\rho, \varphi)$ parameterize the $(1,2)$-plane in space. The normalization has been chosen such that odd integer values of $\Phi$ describe a center vortex, i.e. any Wilson loop $W$ in the $(1,2)$-plane encircling the origin takes the value $W=-1$. The magnetic field associated with (11) is

$$
\overline{\boldsymbol{B}}^{a}(\boldsymbol{x})=\delta^{a 3} \frac{2 \pi \Phi}{g} \delta\left(x_{1}\right) \delta\left(x_{2}\right) \hat{\boldsymbol{e}}_{z}
$$

Our starting point for the evaluation of the one-loop energy density is the Yang-Mills Hamiltonian in Weyl gauge $\left(A_{0}=0\right)$ in the presence of an external current

$$
H=\int \mathrm{d}^{3} x\left[\frac{1}{2} E_{i}^{a} E_{i}^{a}+\frac{1}{2} B_{i}^{a} B_{i}^{a}-j_{i}^{a} A_{i}^{a}\right] .
$$

By decomposing the gauge field $A_{i}^{a}$ into the vortex background field $\bar{A}_{i}^{a}$ and fluctuations $\eta_{i}^{a}$,

$$
A_{i}^{a}=\bar{A}_{i}^{a}+\eta_{i}^{a}
$$

dropping terms of higher order than quadratic in the fluctuations $\eta$ (one-loop approximation), adjusting the external current such as to induce the background field $\overline{\boldsymbol{A}}^{a}$ at the classical level, i.e.

$$
\bar{D}_{i}^{a b} \bar{F}_{i j}^{b}=-j_{j}^{a}, \quad \bar{D}_{i}^{a b}=\delta^{a b} \partial_{i}+g f^{a b c} \bar{A}_{i}^{c}
$$

and finally choosing the background gauge

$$
\bar{D}_{i}^{a b} \eta_{i}^{b}=0
$$


for the fluctuations, one obtains the Hamiltonian (in the "coordinate" representation)

$$
\begin{aligned}
H & =H^{\mathrm{cl}}+H^{(1)} \quad \text { with } \\
H^{\mathrm{cl}} & =\frac{1}{2} \int \mathrm{d}^{3} x\left[\bar{B}_{i}^{a} \bar{B}_{i}^{a}-2 j_{i}^{a} \bar{A}_{i}^{a}\right] \\
H^{(1)} & =\frac{1}{2} \int \mathrm{d}^{3} x\left[-\frac{\delta^{2}}{\left(\delta \eta_{i}^{a}\right)^{2}}+\eta_{i}^{a} \hat{O}_{i j}^{a b} \eta_{j}^{b}\right] .
\end{aligned} \text { and }
$$

Here the operator $\hat{O}$ reads

$$
\hat{O}_{i j}^{a b}=-\delta_{i j}\left(\bar{D}^{2}\right)^{a b}+2 g f^{a b c} \epsilon_{i j k} \bar{B}_{k}^{c},
$$

where $\left(\bar{D}^{2}\right)^{a b}=\bar{D}_{i}^{a c} \bar{D}_{i}^{c b}$. In this approximation, the ground state wave functional takes the Gaussian form

$$
\Psi[\eta]=\exp \left(-\frac{1}{2} \int \eta K \eta\right)
$$

with the spectral representation for the covariance $K$

$$
K_{i j}^{a b}(\boldsymbol{x}, \boldsymbol{y})=\sum_{N}\left(\phi^{\dagger}\right)_{i}^{a}(N, \boldsymbol{x}) \sqrt{O(N)} \phi_{j}^{b}(N, \boldsymbol{y}) .
$$

Here $\phi(N)$ denotes the eigenfunctions of $\hat{O}$, cf. (8),

$$
\hat{O} \phi(N)=O(N) \phi(N)
$$

where $N$ denotes a (not necessarily enumerable) complete set of quantum numbers. Furthermore, the eigenfunctions $\phi(N)$ have to fulfill the "background gauge" condition

$$
\bar{D} \phi(N)=0,
$$

such that the wave functional $\Psi$ fulfills Gauss' law,

$$
\left(\bar{D}_{i}^{a b}+g f^{a b c} \eta_{i}^{c}\right) \frac{\delta}{\delta \eta_{i}^{b}} \Psi[\eta]=0
$$

to the desired order, namely $\mathcal{O}\left(g^{0}\right)$, and is an eigenfunctional of the Hamiltonian (77) with eigenvalue

$$
E=E^{\mathrm{cl}}+\frac{1}{2} \int \mathrm{d}^{3} x \underbrace{\left[\sum_{N}\left(\phi^{\dagger}\right)_{i}^{a}(N, x) \sqrt{O(N)} \phi_{i}^{a}(N, x)\right]}_{K_{i i}^{a a}(x, x)} \equiv \int \mathrm{d}^{3} x e(x),
$$


where $e(x)$ denotes the energy density. To calculate $e(x)$, the set of eigenfunctions $\phi$ must be specified. As a first step, it is useful to reduce the problem by decomposing the functions $\phi($ and the quantum numbers $N$ ) as

$$
\phi_{j}^{b}(N, x)=u_{j}(i) e^{b}(a) f_{M}\left(x_{1}, x_{2}\right) \exp \left(\mathrm{i} k x_{3}\right) \exp (\mathrm{i} m \varphi), \quad k \in \mathbb{R}, m \in \mathbb{Z}
$$

with polar color and Lorentz unit vectors

$$
\begin{aligned}
& e(1)=(1 / \sqrt{2}, \mathrm{i} / \sqrt{2}, 0), \quad e(2)=(1 / \sqrt{2},-\mathrm{i} / \sqrt{2}, 0), e(3)=(0,0,1), \\
& u(1)=(1 / \sqrt{2}, \mathrm{i} / \sqrt{2}, 0), u(2)=(1 / \sqrt{2},-\mathrm{i} / \sqrt{2}, 0), u(3)=(0,0,1),
\end{aligned}
$$

in terms of which the eigenvalue equation (11) reduces to

$$
\hat{B}(a, i, m) f_{M}=B(M, a, i, m) f_{M}
$$

where the operator $\hat{B}$ is defined by

$$
\hat{B}(a, i, m)=-\frac{1}{\rho} \frac{\mathrm{d}}{\mathrm{d} \rho} \rho \frac{\mathrm{d}}{\mathrm{d} \rho}+\frac{(m-\lambda(a) \Phi)^{2}}{\rho^{2}}-2 \lambda(i) \lambda(a) 2 \pi \Phi \delta\left(x_{1}\right) \delta\left(x_{2}\right)
$$

and its eigenvalues $B(M, a, i, m)$ are related to the ones of $\hat{O}$, cf. (요), by

$$
O(N)=B(M, a, i, m)+k^{2}
$$

Note that the color quantum numbers $a$ and the Lorentz quantum numbers $i$ enter via the eigenvalues $\lambda$ corresponding to the vectors (16), namely

$$
\lambda(1)=1 \quad \lambda(2)=-1 \quad \lambda(3)=0 .
$$

The additional constraint on the $f_{M}$ imposed by Gauss' law, eq. (13), will be dealt with later. As the operator $\hat{B}$, cf. (18), is not well defined on the $z$-axis, we restrict space to $\mathbb{R}^{3} \backslash\{z-$ axis $\}$ and take the delta functions into account by adopting appropriate boundary conditions.

At this stage, it is useful to introduce a scale $\bar{M}$ which has the dimension of a mass. This allows us to make eigenvalue equations dimensionless and, when we determine boundary conditions, we make an expansion of functions for distances to the $z$-axis small compared to the scale $1 / \bar{M}$. With this scale, we define a new variable $x:=\rho \bar{M}$ and obtain a dimensionless operator

$$
\hat{C}=\frac{1}{\bar{M}^{2}} \hat{B}=-\frac{1}{x} \frac{\mathrm{d}}{\mathrm{d} x} x \frac{\mathrm{d}}{\mathrm{d} x}+\frac{(m-\lambda(a) \Phi)^{2}}{x^{2}} .
$$


For a complete definition of the set of functions $f_{M}$, a set of boundary conditions in $L^{2}\left(\mathbb{R}_{>0}, x \mathrm{~d} x\right)$ must be specified for $x \rightarrow 0$, such that $\hat{C}$ is self-adjoint. In order to systematically construct all self-adjoint extensions, we initially restrict the domain $D(\hat{C})$ to $D(\hat{C})=C_{c}^{\infty}\left(\mathbb{R}_{>0}\right)$ on which $\hat{C}$ is a well defined symmetric operator, i.e.

$$
\langle f \mid \hat{C} g\rangle=\langle\hat{C} f \mid g\rangle \quad \text { for all } f, g \in D(\hat{C}) .
$$

Self-adjointness, by contrast, means more; namely, that $\hat{C}$ is equal to its adjoint $\hat{C}^{\dagger}$, defined as follows. Let $D\left(\hat{C}^{\dagger}\right)$ be the set of functions $g$ for which $h \in L^{2}\left(\mathbb{R}_{>0}, x \mathrm{~d} x\right)$ exists such that

$$
\langle g \mid \hat{C} f\rangle=\langle h \mid f\rangle \quad \text { for all } f \in D(\hat{C}) .
$$

Then, for each $g \in D\left(\hat{C}^{\dagger}\right)$, one defines $\hat{C}^{\dagger} g=h$. As $D(\hat{C})=C_{c}^{\infty}\left(\mathbb{R}_{>0}\right)$, one sees that in the sense of weak derivatives $-\frac{1}{x} \frac{\mathrm{d}}{\mathrm{d} x} x \frac{\mathrm{d}}{\mathrm{d} x} g$ is locally in $L^{2}\left(\mathbb{R}_{>0}, x \mathrm{~d} x\right)$ and therefore also locally in $L^{1}\left(\mathbb{R}_{>0}, x \mathrm{~d} x\right)$. From this one can conclude that $\left(x g^{\prime}\right)^{\prime}$ is locally in $L^{2}\left(\mathbb{R}_{>0}, \mathrm{~d} x\right)$ with the usual measure and, with Sobolev's lemma [15], that $g^{\prime}$ is absolute continuous. This allows an integration by parts in (23) which gives

$$
\begin{aligned}
\hat{C}^{\dagger} & =-\frac{1}{x} \frac{\mathrm{d}}{\mathrm{d} x} x \frac{\mathrm{d}}{\mathrm{d} x}+\frac{\delta^{2}}{x^{2}} \\
D\left(\hat{C}^{\dagger}\right) & =\left\{f \in L^{2}\left(\mathbb{R}_{>0}, x \mathrm{~d} x\right) \mid f^{\prime} \text { absolute continuous, } \hat{C}^{\dagger} f \in L^{2}\left(\mathbb{R}_{>0}, x \mathrm{~d} x\right)\right\},
\end{aligned}
$$

where the derivatives are now ordinary derivatives and we introduced $\delta=|m-\lambda(a) \Phi|$. Note that $D(\hat{C}) \subset D\left(\hat{C}^{\dagger}\right)$ as it should be for the symmetric operator $\hat{C}$. In the following, we construct all self-adjoint extensions of $\hat{C}$. In the cases where we obtain more than one self-adjoint extension, which corresponds to different possible boundary conditions on the $z$-axis, the choice of a definite self-adjoint extension is a question of the physical situation one wishes to describe; mathematically, all choices are equally valid.

\section{B. Self-adjoint extensions}

To determine self-adjoint extensions of $\hat{C}$, it is necessary to consider the deficiency spaces $\mathcal{H}_{ \pm}$ of $\hat{C}$, i.e. the spaces of zero modes of the operators $\hat{C}^{\dagger} \mp \mathrm{i}$, with their respective dimensions (deficiency indices) $n_{ \pm}[15]$. The operator $\hat{C}$ is called essentially self-adjoint if $n_{+}=n_{-}=0$, 
thus admitting a unique self-adjoint extension given by its closure. With $f \in D\left(\hat{C}^{\dagger}\right)$, cf. (25), and

$$
\hat{C}^{\dagger} f= \pm \mathrm{i} f
$$

one can conclude that $f \in C_{c}^{\infty}\left(\mathbb{R}_{>0}\right)$. Two linearly independent solutions of $\hat{C}^{\dagger} f=$ if are $I_{\delta}\left(\frac{1}{\sqrt{2}}(1-i) x\right)$ and $K_{\delta}\left(\frac{1}{\sqrt{2}}(1-i) x\right)$, where $K_{\delta}$ and $I_{\delta}$ denote the modified Bessel functions. With the condition $f \in D\left(\hat{C}^{\dagger}\right)$ only the solution

$$
f_{+}(x)=K_{\delta}\left(\frac{1}{\sqrt{2}}(1-i) x\right)
$$

for $0 \leq \delta<1$ remains. Therefore

$$
\begin{array}{ll}
\mathcal{H}_{+}=\left\{c f_{+} \mid c \in \mathbb{C}\right\} & \text { for } 0 \leq \delta<1, \\
\mathcal{H}_{+}=\emptyset & \text { for } \delta \geq 1 .
\end{array}
$$

Correspondingly, we obtain

$$
\begin{array}{ll}
\mathcal{H}_{-}=\left\{c f_{-} \mid c \in \mathbb{C}\right\} & \text { for } 0 \leq \delta<1, \\
\mathcal{H}_{-}=\emptyset & \text { for } \delta \geq 1
\end{array}
$$

with

$$
f_{-}(x)=K_{\delta}\left(\frac{1}{\sqrt{2}}(1+\mathrm{i}) x\right) .
$$

As already discussed above, this means that there exists a unique self-adjoint extension for $\delta \geq 1$.

For $0 \leq \delta<1$ the deficiency indices are equal: $n_{+}=n_{-}=1$, such that there is a one parameter family of self-adjoint extensions $\hat{C}_{\alpha}(\alpha \in(-\pi, \pi])$ which are in one-to-one correspondence to isometries $U_{\alpha}: f_{+} \mapsto \exp (\mathrm{i} \alpha) f_{-}$from $\mathcal{H}_{+}$to $\mathcal{H}_{-}$in the following way:

$$
\begin{aligned}
D\left(\hat{C}_{\alpha}\right) & =\left\{f+c\left(f_{+}+U_{\alpha} f_{+}\right) \mid f \in D(\overline{\hat{C}}), c \in \mathbb{C}\right\} \\
\hat{C}_{\alpha}\left(f+c\left(f_{+}+U_{\alpha} f_{+}\right)\right) & =\hat{C} f+c\left(\mathrm{i} f_{+}-\mathrm{i} U_{\alpha} f_{+}\right) .
\end{aligned}
$$

In Appendix $\mathrm{A}$ it is shown that the functions $f \in D(\overline{\hat{C}})$ in the closure of $\hat{C}$ are absolute continuous close to the origin and fulfill $\lim _{x \rightarrow 0} x^{-\delta} f(x)=0$. As the function $f_{+}$is singular at the origin, the boundary conditions at $x=0$ is determined by the behavior of this function. In the following, we determine these boundary conditions for the cases $0<\delta<1$ and $\delta=0$ separately. 
- $0<\delta=|m-\lambda(a)|<1$

Expanding $f \in D\left(\hat{C}_{\alpha}\right)$ for small $x$, one obtains

$$
f(x)=A\left(x^{\delta}+\beta(\alpha) x^{-\delta}\right)+\mathrm{o}\left(x^{\delta}\right), \text { as } x \rightarrow 0
$$

for a constant $A \in \mathbb{C}$ and with the one-to-one map

$$
\beta(\alpha):=\frac{2^{2 \delta} \Gamma(\delta)}{\Gamma(-\delta)} \frac{\cos \left(\frac{\alpha}{2}-\frac{\pi}{4} \delta\right)}{\cos \left(\frac{\alpha}{2}+\frac{\pi}{4} \delta\right)} .
$$

Since $\hat{C} J_{\delta}(\kappa x)=\kappa^{2} J_{\delta}(\kappa x)$ and $\hat{C} N_{\delta}(\kappa x)=\kappa^{2} N_{\delta}(\kappa x)$, one can make the following ansatz for the scattering states,

$$
A J_{\delta}(\kappa x)+B N_{\delta}(\kappa x)
$$

and again expand for small $x$. Comparing this with (31) yields the condition

$$
\frac{A}{B}=\frac{\cos \left(\frac{\alpha}{2}+\frac{\pi}{4} \delta\right)}{\kappa^{2 \delta} \cos \left(\frac{\alpha}{2}-\frac{\pi}{4} \delta\right) \sin (\delta \pi)}-\cot (\delta \pi) .
$$

As bound state, only the solution $K_{\delta}$ is admissible, since $\hat{C} K_{\delta}(\kappa x)=-\kappa^{2} K_{\delta}(\kappa x)$, and $K_{\delta}$ is square-integrable. If one expands $K_{\delta}$ for small $x$ and again compares with (31), one notices that $K_{\delta}$ with

$$
\kappa=\left(\frac{\cos \left(\frac{\alpha}{2}+\frac{\pi}{4} \delta\right)}{\cos \left(\frac{\alpha}{2}-\frac{\pi}{4} \delta\right)}\right)^{\frac{1}{2 \delta}}
$$

is a possible eigenfunction, if

$$
\frac{\cos \left(\frac{\alpha}{2}+\frac{\pi}{4} \delta\right)}{\cos \left(\frac{\alpha}{2}-\frac{\pi}{4} \delta\right)}>0
$$

is satisfied.

- $\delta=|m-\lambda(a)|=0$

Expanding $f \in D\left(\hat{C}_{\alpha}\right)$ for small $x$, one obtains

$$
f(x)=A\left(\ln \left(\frac{x}{2}\right)+\beta(\alpha)\right)+\mathrm{o}\left(x^{0}\right), \text { as } x \rightarrow 0
$$

for a constant $A \in \mathbb{C}$ and with the one-to-one map

$$
\beta(\alpha):=\gamma-\frac{\pi}{4} \tan \left(\frac{\alpha}{2}\right)
$$


where $\gamma$ is Euler's constant. For the scattering states, one makes, as above, the ansatz

$$
A J_{0}(\kappa x)+B N_{0}(\kappa x)
$$

which, after expanding for small $x$ and comparing with (32), yields the condition

$$
\frac{A}{B}=-\frac{2}{\pi} \ln (\kappa)-\frac{1}{2} \tan \left(\frac{\alpha}{2}\right) .
$$

The possible bound state is

$$
K_{0}(\kappa x),
$$

provided that

$$
\kappa=\exp \left(-\frac{\pi}{4} \tan \left(\frac{\alpha}{2}\right)\right)
$$

is satisfied; correspondingly, one has the eigenvalue

$$
-\kappa^{2}=-\exp \left(-\frac{\pi}{2} \tan \left(\frac{\alpha}{2}\right)\right) .
$$

For $\alpha=\pi$ there is no bound state and the solutions are regular at the origin $(B=0)$.

\section{Gauss' law and choice of self-adjoint extension}

In the previous section, for the cases $0 \leq \delta \equiv|m-\lambda(a) \Phi|<1$ a whole family of self-adjoint

extensions of the operator $\hat{C}$ was obtained, labeled by one continuous parameter $\alpha$; each value corresponds to a different choice of boundary conditions reflecting different physical situations. In this section, a definite choice for the parameter $\alpha$ will be motivated.

The operator $\hat{B}$, cf. (18), can be interpreted physically as the Hamiltonian of a particle with effective angular momentum (grand spin) $\delta=m-\lambda(a) \Phi$ in a potential $-2 \lambda(i) \lambda(a) 2 \pi \Phi \delta\left(x_{1}\right) \delta\left(x_{2}\right)$. Therefore, a bound state can only arise if the potential term is negative, i.e. $\lambda(i) \lambda(a)>0$ for positive flux $\Phi>0$. This is the case for $\lambda(i)=\lambda(a)=1$ and for $\lambda(i)=\lambda(a)=-1$. It will now be demonstrated that Gauss' law forbids bound states even in these two cases.

In order to fulfill Gauss' law, it is necessary to restrict the set of functions $\phi(N)$ in each subspace of definite eigenvalue $O(N)$ to those linear combinations which satisfy eq. (12). Since the corresponding operator $\bar{D}_{i}^{a b}$ does not mix functions $\phi(N)$ with a different color quantum number $a$, such linear combinations can be constructed for each $a$ separately. The 
same is true for the momentum in 3-direction $k$ and the angular momentum quantum number $m$. Concentrating on the bound state solutions in the case $\lambda(a)=\lambda(i)=1$, one has, using the decomposition (15),

$$
\bar{D}_{j}^{a b} \phi_{j}^{b}(N) \stackrel{!}{=} 0 \Rightarrow\left(\rho \partial_{\rho}-m+\Phi\right) f_{M}=0
$$

which for the bound state solutions $f_{M}(\rho)=K_{|m-\Phi|}(\kappa \rho)$ would require

$$
(|m-\Phi|-m+\Phi) K_{|m-\Phi|}(\kappa \rho)-\kappa \rho K_{|m-\Phi|+1}(\kappa \rho)=0
$$

For this to be true, both coefficients must vanish; in particular, $\kappa=0$, which contradicts the condition $\kappa>0$. Therefore, there can be no bound state for $\lambda(a)=\lambda(i)=1$. The same is true in the case $\lambda(a)=\lambda(i)=-1$. For $\Phi \in \mathbb{Z}$, this singles out a definite choice of the self-adjoint extension, namely $\alpha=\pi$, since this is the only choice which yields no bound state. In this case, $B=0$, i.e. the functions are regular at the origin. It should be noted that for non-integer flux $\Phi$, in general there are several possibilities with no bound states. In the following, however, regularity at the origin $(B=0)$ will be demanded even for non-integer flux.

From the physical point of view, this outcome is natural. Gauss' law enforces gauge invariance; gauge invariance in particular implies that infinitely thin integer fluxes, which are pure gauges in the theory without quark degrees of freedom, should be unobservable. Therefore, the gluonic excitation spectrum in the presence of such a background field should be equivalent to the free case. However, it should be emphasized that other interpretations, corresponding to other choices of self-adjoint extension, are possible and should not a priori be dismissed as invalid; e.g. the thin vortex fluxes treated here may be used as idealizations of physical, thick fluxes which are not pure gauges. Then, a different choice of self-adjoint extension may be useful to mimic the effects of vortex thickness.

\section{Energy density of a vortex to order $g^{0}$}

For the particular choice of self-adjoint extension adopted above, in the sectors with $\lambda(a)=$ \pm 1 the energy density associated with a vortex differs from the one in the vacuum by

$$
e_{ \pm}=2 \frac{\bar{M}^{2}}{2 \pi} \int_{0}^{\infty} \kappa \mathrm{d} \kappa \frac{1}{2 \pi} \int_{-\infty}^{\infty} \mathrm{d} k \sum_{m=-\infty}^{\infty} \sqrt{(\kappa \bar{M})^{2}+k^{2}}\left[\left(J_{|m \mp \Phi|}(\kappa x)\right)^{2}-\left(J_{|m|}(\kappa x)\right)^{2}\right]
$$


(for $x>0$ ) where the prefactor 2 comes from the two physical polarizations allowed by the Gauss'law constraint. In the sector with $\lambda(a)=0$, the energy density (compared with the one of the vacuum) obviously vanishes.

Let us first consider an integer-valued flux $\Phi \in \mathbb{N}_{0}, \lambda(a) \pm 1$. For $\Phi \in \mathbb{N}_{0}$, one has

$$
\sum_{m=-\infty}^{\infty}\left(J_{|m|}(\kappa x)\right)^{2}=1 \text { and } \sum_{m=-\infty}^{\infty}\left(J_{|m \mp \Phi|}(\kappa x)\right)^{2}=1 .
$$

Thus, the energy density (35) vanishes for all $x>0$ and therefore

$$
e(\rho)=0 \text { for } \rho>0
$$

The invisibility of thin integer vortex fluxes $\Phi$, which represent pure gauge backgrounds, thus becomes manifest.

Let us now consider fluxes $\Phi \in(0,1)$. Consider first the energy density (35) in the sector given by $\lambda(a)=1$ : Rewriting the square root in eq. (35) by a proper-time integral

$$
\sqrt{a}=-\lim _{s_{\min } \rightarrow 0} \int_{s_{\min }}^{\infty} \mathrm{d} s(\pi s)^{-1 / 2} \frac{\mathrm{d}}{\mathrm{d} s} \mathrm{e}^{-s a},
$$

where $s_{\min }$ is an (ultraviolet) proper-time cut-off we find

$$
\begin{aligned}
e_{+}= & -2 \bar{M}^{2} \int_{s_{\min }}^{\infty} \mathrm{d} s(\pi s)^{-1 / 2} \frac{\mathrm{d}}{\mathrm{d} s} \frac{1}{2 \pi} \int_{0}^{\infty} \kappa \mathrm{d} \kappa \frac{1}{2 \pi} \int_{-\infty}^{\infty} \mathrm{d} k \sum_{m=-\infty}^{\infty} \mathrm{e}^{-s\left((\bar{M} \kappa)^{2}+k^{2}\right)} \\
& \times\left[\left(J_{|m-\Phi|}(\kappa x)\right)^{2}-\left(J_{|m|}(\kappa x)\right)^{2}\right]
\end{aligned}
$$

The integrals over $k$ and $\kappa$ can be performed by using the relation

$$
\int_{0}^{\infty} \kappa \mathrm{d} \kappa \mathrm{e}^{-s(\bar{M} \kappa)^{2}} J_{\nu}(\kappa x)^{2}=\frac{1}{2 s \bar{M}^{2}} \mathrm{e}^{\frac{x^{2}}{2 s \bar{M}^{2}}} I_{\nu}\left(\frac{x^{2}}{2 s \bar{M}^{2}}\right), \nu=|m-\Phi| \text { or } \nu=|m|,
$$

cf. eq. 6.633 .2 in [16], yielding

$e_{+}=-2 \bar{M}^{2} \int_{s_{\text {min }}}^{\infty} \mathrm{d} s(\pi s)^{-1 / 2} \frac{\mathrm{d}}{\mathrm{d} s} \frac{1}{2 \pi} \sqrt{\frac{\pi}{s}} \sum_{m=-\infty}^{\infty} \frac{1}{2 \pi} \frac{1}{2 s \bar{M}^{2}} \mathrm{e}^{-\frac{x^{2}}{2 s \bar{M}^{2}}}\left[I_{|m-\Phi|}\left(\frac{x^{2}}{2 s \bar{M}^{2}}\right)-I_{|m|}\left(\frac{x^{2}}{2 s \bar{M}^{2}}\right)\right]$.

By substituting $t=\frac{x^{2}}{2 s M^{2}}=\frac{\rho^{2}}{2 s}$, one arrives at

$$
\begin{aligned}
e_{+} & =-2 \int_{t_{\max }}^{0} \mathrm{~d} t \sqrt{\frac{2 t}{\rho^{2}}} \frac{\mathrm{d}}{\mathrm{d} t} \frac{1}{2 \pi} \sqrt{\frac{2 t}{\rho^{2}}} \sum_{m=-\infty}^{\infty} \frac{1}{2 \pi} \frac{t}{\rho^{2}} \mathrm{e}^{-t}\left[I_{|m-\Phi|}(t)-I_{|m|}(t)\right] \\
& =\frac{2}{2 \pi^{2} \rho^{4}} \int_{0}^{t_{\max }} \mathrm{d} t t^{1 / 2} \frac{\mathrm{d}}{\mathrm{d} t} t^{3 / 2} \mathrm{e}^{-t}\left(I_{\Phi}(t)-I_{0}(t)+\sum_{m=1}^{\infty}\left[I_{m-\Phi}(t)+I_{m+\Phi}(t)-2 I_{m}(t)\right]\right) .
\end{aligned}
$$


After inserting the integral representation [16]

$$
I_{\nu}(t)=\frac{1}{\pi} \int_{0}^{\pi} \mathrm{e}^{t \cos (\theta)} \cos (\nu \theta) \mathrm{d} \theta-\frac{\sin (\nu \pi)}{\pi} \int_{0}^{\infty} \mathrm{e}^{-t \cosh (z)-\nu z} \mathrm{~d} z
$$

into $e_{+}$, the first terms of the integral representations of the different modified Bessel functions cancel, so that the only non-vanishing contribution reads

$$
\begin{aligned}
e_{+} & =-\frac{2}{2 \pi^{2} \rho^{4}} \int_{0}^{t_{\max }} \mathrm{d} t t^{1 / 2} \frac{\mathrm{d}}{\mathrm{d} t} t^{3 / 2} \mathrm{e}^{-t} \frac{1}{\pi}\left(\int_{0}^{\infty} d z \mathrm{e}^{-t \cosh (z)} \sin (\Phi \pi) \mathrm{e}^{-\Phi z}\right. \\
& \left.+\sum_{m=1}^{\infty} \int_{0}^{\infty} \mathrm{d} z \mathrm{e}^{-t \cosh (z)}\left(-\cos (m \pi) \sin (\Phi \pi) \mathrm{e}^{-(m-\Phi) z}+\cos (m \pi) \sin (\Phi \pi) \mathrm{e}^{-(m+\Phi) z}\right)\right) \\
& =-\frac{2}{2 \pi^{2} \rho^{4}} \frac{\sin (\Phi \pi)}{\pi} \int_{0}^{t_{\max }} \mathrm{d} t t^{1 / 2} \frac{d}{d t} t^{3 / 2} \int_{0}^{\infty} \mathrm{d} z \mathrm{e}^{-t(\cosh (z)+1)} \frac{\cosh \left(z\left[\frac{1}{2}-\Phi\right]\right)}{\cosh \left(\frac{z}{2}\right)} .
\end{aligned}
$$

Partially integrating with respect to $t$ and noting that the surface term vanishes in the limit $t_{\max } \rightarrow \infty$, one obtains

$$
e_{+}=\frac{2}{\left(2 \pi \rho^{2}\right)^{2}} \frac{\sin (\Phi \pi)}{\pi} \int_{0}^{t_{\max }} \mathrm{d} t t \int_{0}^{\infty} \mathrm{d} z \mathrm{e}^{-t(\cosh (z)+1)} \frac{\cosh \left(z\left[\frac{1}{2}-\Phi\right]\right)}{\cosh \left(\frac{z}{2}\right)} .
$$

For $\rho>0$ the limit $s_{\min } \rightarrow 0$ implies $t_{\max } \rightarrow \infty$. Then the integration over $t$ can be carried out with a finite result,

$$
\begin{aligned}
e_{+} & =\frac{2}{\left(2 \pi \rho^{2}\right)^{2}} \frac{\sin (\Phi \pi)}{\pi} \int_{0}^{\infty} \mathrm{d} z \frac{1}{(\cosh (z)+1)^{2}} \frac{\cosh \left(z\left[\frac{1}{2}-\Phi\right]\right)}{\cosh \left(\frac{z}{2}\right)} \\
& =\frac{2}{\left(2 \pi \rho^{2}\right)^{2}} \frac{\sin (\Phi \pi)}{\pi} 4 \int_{0}^{\infty} \mathrm{d} z \frac{\mathrm{e}^{z(1 / 2-\Phi)}+\mathrm{e}^{-z(1 / 2-\Phi)}}{\left(\mathrm{e}^{z / 2}+\mathrm{e}^{-z / 2}\right)\left(\mathrm{e}^{z}+\mathrm{e}^{-z}+2\right)^{2}}
\end{aligned}
$$

Substituting $x=\mathrm{e}^{z}$ yields

$$
\begin{aligned}
e_{+} & =\frac{2}{\left(\pi \rho^{2}\right)^{2}} \frac{\sin (\Phi \pi)}{\pi} \int_{1}^{\infty} \frac{\mathrm{d} x}{x} \frac{x^{1 / 2-\Phi}+x^{\Phi-1 / 2}}{\left(x^{1 / 2}+x^{-1 / 2}\right)\left(x+x^{-1}+2\right)^{2}} \\
& =\frac{2}{\left(\pi \rho^{2}\right)^{2}} \frac{\sin (\Phi \pi)}{\pi} \int_{1}^{\infty} \mathrm{d} x \frac{x^{2-\Phi}+x^{\Phi+1}}{(x+1)^{5}} \\
& =\frac{2}{\left(\pi \rho^{2}\right)^{2}} \frac{\sin (\Phi \pi)}{\pi} \frac{\Gamma(3-\Phi) \Gamma(2+\Phi)}{\Gamma(5)} \\
& =\frac{1}{\rho^{4}} \frac{1}{12 \pi^{2}} \Phi\left(1-\Phi^{2}\right)(2-\Phi) \text { for } \Phi \in(0,1) .
\end{aligned}
$$




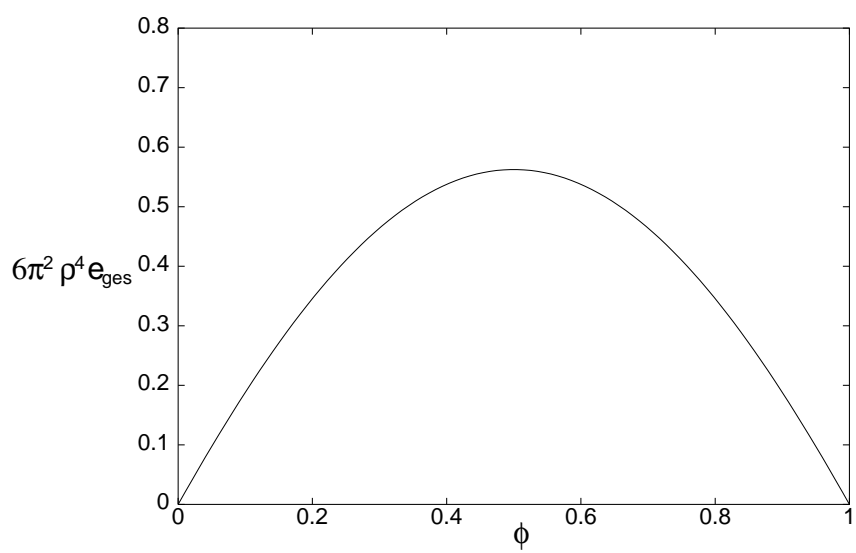

FIG. 1: Energy density $e_{\text {ges }}$ as a function of magnetic flux $\phi$.

For $e_{-}$, i.e. $\lambda(a)=-1$, one obtains the same result in a completely analogous fashion. Furthermore, according to (35) the energy density is invariant under integer shifts in $\Phi$. The total energy density

$$
e_{\text {ges }}=e_{+}+e_{-}=\frac{1}{\rho^{4}} \frac{1}{6 \pi^{2}} \phi\left(1-\phi^{2}\right)(2-\phi) \quad \text { for } \quad \Phi=N+\phi, N \in \mathbb{N}_{0}, \phi \in[0,1)
$$

is depicted in Fig. 1 . The fact that the energy density is proportional to $\frac{1}{\rho^{4}}$ is natural on dimensional grounds. Furthermore, for integer-valued $\Phi$, the fluctuations are equivalent to the ones in the vacuum, as expected for background fields representing pure gauges in the theory without quarks, i.e. in the presence of fluctuating fields in the adjoint representation of the gauge group only. Such fluctuations are not sensitive to a thin integer flux vortex.

\section{FERMIONS IN THE VORTEX BACKGROUND FIELD}

In this section we calculate the energy density of the quark fluctuations in a vortex background field. The Hamiltonian of the quarks $\psi(x)$ in the gauge field $A_{\mu}(x)$ satisfying the Weyl gauge $A_{0}(x)=0$ is given by

$$
H=\int \mathrm{d}^{3} x \psi^{\dagger}(\boldsymbol{x}) h(\boldsymbol{x}) \psi(\boldsymbol{x})
$$

\footnotetext{
${ }^{1}$ This result agrees with the one obtained in ref. [14]. However, there the freedom in the choice of the self-adjoint extension was not discussed and a particular choice was made ad hoc.
} 
where

$$
h(\boldsymbol{x})=\boldsymbol{\alpha}(\boldsymbol{p}+g \boldsymbol{A})+\beta m
$$

is the Dirac Hamiltonian with $\boldsymbol{\alpha}, \beta$ denoting the usual Dirac matrices. In the following we will, for simplicity, consider massless quarks $(m=0)$, which is a good approximation for light quark flavours. Furthermore, we will use the Weyl representation of the Dirac matrices, such that the Dirac operator, eq. (43), becomes block diagonal,

$$
h=\left(\begin{array}{cc}
D & 0 \\
0 & -D
\end{array}\right), \quad D=\boldsymbol{\sigma}(\boldsymbol{p}+g \boldsymbol{A})
$$

where $\boldsymbol{\sigma}$ are the usual Pauli spin matrices. For the gauge potential of the vortex field defined by eq. (11) the operator (44) is diagonal in color space

$$
D_{a b}=\delta_{a b} D(a), \quad D(a)=\boldsymbol{\sigma}\left(-\mathrm{i} \boldsymbol{\nabla}+\frac{\lambda(a)}{2} \frac{\Phi}{\rho} \boldsymbol{e}_{\varphi}\right)
$$

where $\lambda(a)=1,-1$ for $a=1,2$. Due to the geometry of the vortex field, it is convenient to use cylindrical coordinates $(\rho, \varphi, z)$ in which the operator, eq. (45), reads

$$
D(a)=\left(\begin{array}{cc}
-\mathrm{i} \partial_{z} & \mathrm{ie}^{-\mathrm{i} \varphi}\left(-\partial_{\rho}-\frac{1}{\rho}\left(l+\frac{\lambda(a)}{2} \Phi\right)\right) \\
-\mathrm{i} \mathrm{e}^{\mathrm{i} \varphi}\left(\partial_{\rho}-\frac{1}{\rho}\left(l+\frac{\lambda(a)}{2} \Phi\right)\right) & \mathrm{i} \partial_{z}
\end{array}\right)
$$

where $l=-\mathrm{i} \partial_{\varphi}$ is the orbital angular momentum. It is seen that the vortex field generates an intrinsic angular momentum (color spin) of the quarks

$$
s=\frac{1}{2} \lambda(a) \Phi
$$

For center vortices, where $\Phi=1$, and, since $|\lambda(a)|=1$, the total (grand) angular momentum of the fermions $J=l+s$ becomes half-integer valued, which is reminiscent of a boson-fermion transmutation.

To find the spinor eigenfunctions $u$ of the operator $D(a)$, cf. (46), we make the following ansatz, 


$$
u\left(a, k, m, N^{\prime} ; \boldsymbol{x}\right)=\mathrm{e}^{\mathrm{i} k z}\left(\begin{array}{c}
\mathrm{e}^{\mathrm{i} m \varphi} \chi\left(a, k, m, N^{\prime} ; \rho\right) \\
\mathrm{e}^{\mathrm{i}(m+1) \varphi} \psi\left(a, k, m, N^{\prime} ; \rho\right)
\end{array}\right), k \in \mathbb{R}, m \in \mathbb{Z}
$$

where the quantum number $N^{\prime}$ will be specified further below. With this ansatz, the eigenvalue equation for the operator $D(a)$, eq. (46), reduces to

$$
\left(\begin{array}{cc}
k & \mathrm{i}\left(-\partial_{\rho}-\frac{1+\nu}{\rho}\right) \\
-\mathrm{i}\left(\partial_{\rho}-\frac{\nu}{\rho}\right) & -k
\end{array}\right)\left(\begin{array}{l}
\chi\left(a, k, m, N^{\prime} ; \rho\right) \\
\psi\left(a, k, m, N^{\prime} ; \rho\right)
\end{array}\right)=E\left(a, k, m, N^{\prime}\right)\left(\begin{array}{l}
\chi\left(a, k, m, N^{\prime} ; \rho\right) \\
\psi\left(a, k, m, N^{\prime} ; \rho\right)
\end{array}\right)
$$

where we have introduced the quantum number

$$
\nu:=m+\frac{\lambda(a) \Phi}{2}
$$

of the grand angular momentum $J=l+s$. Note that the eigenvalue equation (49) is equivalent to the Dirac equation in two space-time dimensions for a particle with mass $k$. In the standard fashion, eq. (49) can be reduced to a second order differential equation for the radial functions $\chi, \psi$ which takes the form of Bessel's differential equation. The solutions of the eigenvalue eq. (49) for $E^{2}>k^{2}$ (scattering states) are given by

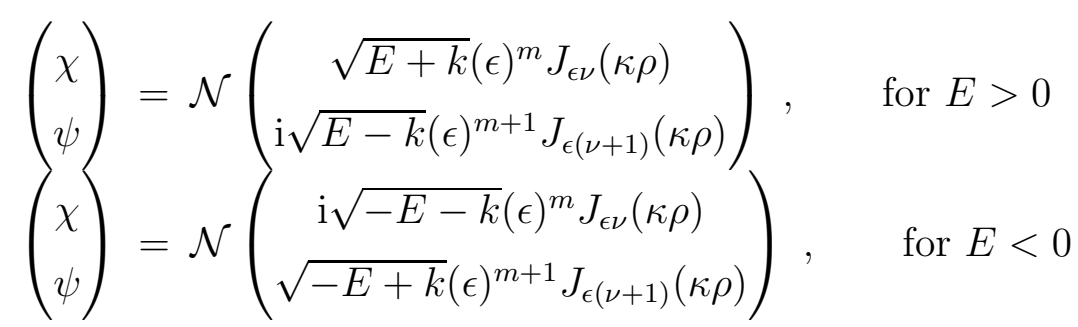

where $\mathcal{N}$ is a normalization constant and $\kappa=\sqrt{E^{2}-k^{2}}$ is the wave number in the plane perpendicular to the center vortex. Furthermore, $J_{\alpha}(x)$ denotes the ordinary Bessel functions and we have introduced the parameter $\epsilon= \pm 1$. The wave functions, eq. (51), are solutions of eq. (49) for both values of this parameter and for the eigenvalues $E= \pm \sqrt{\kappa^{2}+k^{2}}$. For $\nu=n \in \mathbb{Z}$ the solutions with $\epsilon= \pm 1$ are linearly dependent, since the Bessel functions satisfy the relations $J_{-n}(x)=(-1)^{n} J_{n}(x)$; thus, one should take Bessel functions and Neumann functions as linearly independent solutions. For $\nu \notin \mathbb{Z}$, which holds in particular for a center vortex field, for which $\Phi=1$, the solutions with $\epsilon= \pm 1$ are linearly independent, i.e. there are two linearly independent solutions for each eigenvalue. However, requiring the functions to be square integrable on the $z$-axis selects out a single solution (that is, either 
$\epsilon=1$ or $\epsilon=-1)$ except for $-1<\nu<0$. The latter interval includes the case $\nu=-\frac{1}{2}$ which occurs for a center vortex, $\Phi=1$ (when $\lambda(a)=1$ and $m=-1$ or when $\lambda(a)=-1$ and $m=0)$. In this case, both solutions $\epsilon= \pm 1$ are square integrable on the $z$-axis.

In the following, we will investigate the case $-1<\nu<0$ in more detail. The point is that we have to impose appropriate boundary conditions on the $z$-axis, where the vortex field has a singularity, so that a unique solution survives. The boundary conditions have to be chosen in such a way that the operator in eq. (45) becomes self-adjoint. The procedure works analogously to the gluonic case: One restricts the domain of definition of the operator $D(a)$, eq. (45), in a suitable way, such that it becomes symmetric. Then one determines the deficiency spaces of this operator and, from this, all its self-adjoint extensions. The construction of all self-adjoint extensions of the operator in eq. (49) was given in [17, 18]. The extensions are characterized by a parameter $\theta \in[0,2 \pi)$ and defined by the following boundary conditions on the eigenfunctions,

$$
\left(\begin{array}{l}
\chi \\
\psi
\end{array}\right) \rightarrow\left(\begin{array}{c}
\mathrm{i}(\bar{M} \rho)^{\nu} \sin \left(\frac{\pi}{4}+\frac{\theta}{2}\right) \\
(\bar{M} \rho)^{-\nu-1} \cos \left(\frac{\pi}{4}+\frac{\theta}{2}\right)
\end{array}\right) \quad \text { for } \rho \rightarrow 0,
$$

where $\bar{M}$ is a mass scale introduced in order to have dimensionless quantities like $\bar{M} \rho$. The requirement of self-adjointness restricts the choice of the boundary conditions on the vortex axis (z-axis). For the boundary conditions (52), a unique solution follows for each value of $\theta \in[0,2 \pi)$, which in the case of $E>0$ is given by

$$
\begin{array}{r}
\left(\begin{array}{l}
\chi \\
\psi
\end{array}\right)=\mathcal{N}\left[1+(-1)^{m} \sin (2 \mu) \cos (\nu \pi)\right]^{-1 / 2} \\
\left(\begin{array}{c}
\sqrt{E+k}\left[\sin (\mu) J_{\nu}(\kappa \rho)+(-1)^{m} \cos (\mu) J_{-\nu}(\kappa \rho)\right] \\
\mathrm{i} \sqrt{E-k}\left[\sin (\mu) J_{\nu+1}(\kappa \rho)+(-1)^{m+1} \cos (\mu) J_{-(\nu+1)}(\kappa \rho)\right]
\end{array}\right)
\end{array}
$$

where the quantity $\mu$ is related to $\theta$ by

$$
\tan \left(\frac{\pi}{4}+\frac{\theta}{2}\right)=(-1)^{m}\left(\frac{E+k}{E-k}\right)^{1 / 2}\left(\frac{\kappa}{2 k}\right)^{2 \nu+1} \frac{\Gamma(-\nu)}{\Gamma(\nu+1)} \tan (\mu) .
$$

In addition to these scattering states, for $\frac{\pi}{2}<\theta<\frac{3 \pi}{2}$ bound states, i.e. square integrable eigenstates exist [17, 18]. 
Finally, let us remark that for $\nu<-1$ and for $\nu>0$, where the parameter $\epsilon= \pm 1$ is already uniquely determined by requiring square integrability of the wave function on the $z$-axis, the application of the above procedure for finding self-adjoint extensions of the Dirac operator is trivial. In fact, one finds that the deficiency spaces of this operator are empty and that hence this operator is essentially self-adjoint.

In those cases where there is more than one self-adjoint extension, one has to select one on physical grounds. In reference [19] it was shown that only for $\theta= \pm \frac{\pi}{2}$ a vortex on the $z$-axis can be consistently described in the sense that the eigenfunctions of the self-adjoint extensions of the Dirac operator and its square fulfill the same boundary conditions. It is still open which sign of $\theta$ has to be chosen for each flux $\Phi$. The sign of $\theta$ can be fixed by requiring that the energy of the quarks in a vortex field should be a periodic function in the flux $\Phi$ of period 2. This is because fermions should not feel a Dirac string, $\Phi=2$. It turns out that this requires choosing $\theta$ such that the assignments listed in the table below, cf. (56), result. Let us emphasize that this implies different signs for different ranges of flux. The same self-adjoint extension of the Dirac operator was obtained in [20] by demanding that the expectation value of the resulting baryon number

$$
\langle N\rangle=-\frac{1}{2} \sum_{n} \operatorname{sgn}\left(E_{n}\right)
$$

properly transforms under charge conjugation, $\langle N\rangle \rightarrow-\langle N\rangle$, and is a periodic function in the flux $\Phi$ with period 2 .

It turns out that this choice of the parameter $\theta$ corresponds to choosing the minimal irregularity of the eigenfunctions on the vortex axis.

\begin{tabular}{|l|l|l|}
\hline & $0<\Phi<1$ & $1<\Phi<2$ \\
\hline$\lambda(a)=1$ & $\epsilon=1$ for $m=0,1, \ldots$ & $\epsilon=1$ for $m=-1,0, \ldots$ \\
& $\epsilon=-1$ for $m=-1,-2, \ldots$ & $\epsilon=-1$ for $m=-2,-3, \ldots$ \\
\hline$\lambda(a)=-1$ & $\epsilon=1$ for $m=0,1, \ldots$ & $\epsilon=1$ for $m=1,2, \ldots$ \\
& $\epsilon=-1$ for $m=-1,-2, \ldots$ & $\epsilon=-1$ for $m=0,-1, \ldots$ \\
\hline
\end{tabular}

Furthermore, the same choice of self-adjoint extension was obtained in reference [21] by choosing boundary conditions of the Atiyah-Patodi-Singer type for a finite radius of the vortex flux and subsequently letting this radius go to zero. 


\section{A. The energy density of quarks in a vortex background}

In the following, we calculate the energy density of fermion fluctuations in the vortex gauge field. The energy density is defined by

$$
e(\boldsymbol{x})=\sum_{a=1,2} \sum_{\tilde{E}(a, N, n)<0} \tilde{E}(a, N, n)\left(\Psi^{a}\right)^{\dagger}(a, N, n ; \boldsymbol{x}) \Psi^{a}(a, N, n ; \boldsymbol{x})
$$

where $\tilde{E}$ and $\Psi$ denote the energy eigenvalues and eigenfunctions of the Dirac Hamiltonian, eq. (43). Due to the block structure of the Dirac Hamiltonian, these eigenvalues are given by both the positive and negative eigenvalues $E$ appearing in eq. (49), and the energy density becomes

$$
\begin{aligned}
e(\boldsymbol{x})= & \sum_{a=1,2}(\underbrace{\sum_{N, E(a, N)<0} E(a, N) u^{\dagger}(a, N ; \boldsymbol{x}) u(a, N ; \boldsymbol{x})}_{=: e(a, E<0 ; \boldsymbol{x})} \\
& -\underbrace{\left.\sum_{N, E(a, N)>0} E(a, N) u^{\dagger}(a, N ; \boldsymbol{x}) u(a, N ; \boldsymbol{x})\right)}_{=: e(a, E>0 ; \boldsymbol{x})}
\end{aligned}
$$

with $u$ denoting the wave functions corresponding to the energy eigenvalues $E$. We have assumed here that the wave functions are properly normalized (as scattering states),

$$
\int_{0}^{\infty} \rho d \rho u^{\dagger}(a, N, \kappa, \boldsymbol{x}) u\left(a, N, \kappa^{\prime}, \boldsymbol{x}\right)=\frac{1}{\kappa} \delta\left(\kappa-\kappa^{\prime}\right) .
$$

This fixes the normalization constant to

$$
\mathcal{N}=\frac{1}{\sqrt{2|E|}}
$$

We have to choose the eigenfunctions such that they respect the boundary conditions corresponding to the minimal irregularity on the vortex axis (56).

With this choice of the eigenfunctions, one finds after a straightforward calculation the 
energy density for fluxes $0<\Phi<1$,

$$
e_{1}(\Phi ; \boldsymbol{x})=-\frac{1}{2 \pi^{2}} \int_{-\infty}^{\infty} \mathrm{d} k \int_{0}^{\infty} \kappa \mathrm{d} \kappa \sqrt{\kappa^{2}+k^{2}}\left(2 \sum_{m=-\infty}^{\infty} J_{|m+\Phi / 2|}^{2}(\kappa \rho)+J_{-\Phi / 2}^{2}(\kappa \rho)-J_{\Phi / 2}^{2}(\kappa \rho)\right)
$$

while for fluxes $1<\Phi<2$ one obtains

$e_{2}(\Phi ; \boldsymbol{x})=-\frac{1}{2 \pi^{2}} \int_{-\infty}^{\infty} \mathrm{d} k \int_{0}^{\infty} \kappa \mathrm{d} \kappa \sqrt{\kappa^{2}+k^{2}}\left(2 \sum_{m=-\infty}^{\infty} J_{|m+\Phi / 2|}^{2}(\kappa \rho)+J_{\Phi / 2-1}^{2}(\kappa \rho)-J_{1-\Phi / 2}^{2}(\kappa \rho)\right)$.

These two expressions for the energy density coincide for the flux $\Phi=1$, which corresponds to a center vortex. Thus, the energy density as a function of the flux $\Phi$ is continuous at the center vortex flux $\Phi=1$. Furthermore, one also immediately recognizes that the relation $e_{1}(\Phi=0 ; \boldsymbol{x})=e_{2}(\Phi=2 ; \boldsymbol{x})$ holds, which implies that the energy density of a Dirac string, $\Phi=2$, is equal to the energy density of the perturbative vacuum, $\Phi=0$. This is expected, since the Dirac string does not carry observable physical flux. Let us emphasize that this degeneracy in the energy density justifies the self-adjoint continuation chosen above.

Since the energy eigenvalues depend only on the combination $\lambda(a) \Phi$ and summation is carried out over $\lambda(a)= \pm 1$, the energy density is reflexion symmetric, i.e. stays invariant under the replacement $\Phi \rightarrow-\Phi$. Furthermore, as can be read off from the explicit expressions (61), (62), the energy densities for the flux ranges $0<\Phi<1$ and $1<\Phi<2$ are related by

$$
e_{1}(\Phi)=e_{2}(2-\Phi)
$$

We can therefore restrict ourselves to the flux interval $0<\Phi<1$. Subtracting from the energy density of the quark fluctuations in the presence of the vortex the corresponding energy density in the perturbative vacuum, one finds 


$$
\begin{aligned}
\Delta e_{1}(\Phi ; \boldsymbol{x})= & e_{1}(\Phi ; \boldsymbol{x})-e_{1}(\Phi=0 ; \boldsymbol{x}) \\
= & \underbrace{-\frac{2}{2 \pi^{2}} \int_{-\infty}^{\infty} \mathrm{d} k \int_{0}^{\infty} \kappa \mathrm{d} \kappa \sqrt{\kappa^{2}+k^{2}} \sum_{-\infty}^{\infty}\left(J_{|m+\Phi / 2|}^{2}(\kappa \rho)-J_{|m|}^{2}(\kappa \rho)\right)}_{=: A} \\
& \underbrace{-\frac{1}{2 \pi^{2}} \int_{-\infty}^{\infty} \mathrm{d} k \int_{0}^{\infty} \kappa \mathrm{d} \kappa \sqrt{\kappa^{2}+k^{2}}\left(J_{-\Phi / 2}^{2}(\kappa \rho)-J_{\Phi / 2}^{2}(\kappa \rho)\right)}_{=: B}
\end{aligned}
$$

The sums which occur here are identical to the sums which occur in the gluonic part of the energy density. In fact, using the results obtained for the gluonic part, see eq. (35), and replacing $\Phi$ by $\Phi / 2$, one finds for the first term

$$
A=-\frac{1}{6 \pi^{2} \rho^{4}} \frac{\Phi}{2}\left(1-\left(\frac{\Phi}{2}\right)^{2}\right)\left(2-\frac{\Phi}{2}\right) .
$$

Analogous calculations as in the gluon sector yield for the second term

$$
B=-\frac{1}{\pi^{2} \rho^{4}} \int_{0}^{\infty} \mathrm{d} t t^{1 / 2} \frac{\mathrm{d}}{\mathrm{d} t} t^{3 / 2} \mathrm{e}^{-t}\left(I_{-\Phi / 2}(t)-I_{\Phi / 2}(t)\right)
$$

The remaining integral has to be carried out numerically for given vortex flux $\Phi$. Adding this contribution to the gluonic part, we obtain the total energy density of the fluctuating gauge field and of the fermions in the vortex background field. The result is shown in Fig. 2.

\section{DISCUSSION}

We have evaluated the one-loop effective energy density associated with an infinitely thin $\mathrm{SU}(2)$ Yang-Mills vortex, taking into account both gluonic as well as (massless) quark fluctuations, for arbitrary vortex flux $\Phi$. As a function of the latter, center vortices (odd integer $\Phi)$ represent local minima of the effective energy - it is energetically favorable to keep magnetic flux bundled in units corresponding to center elements of the gauge group as opposed to letting magnetic flux disassociate and propagate in lines of arbitrary flux through space. In this sense, the quantum fluctuations tend to stabilize center vortices with respect to local deformations. 


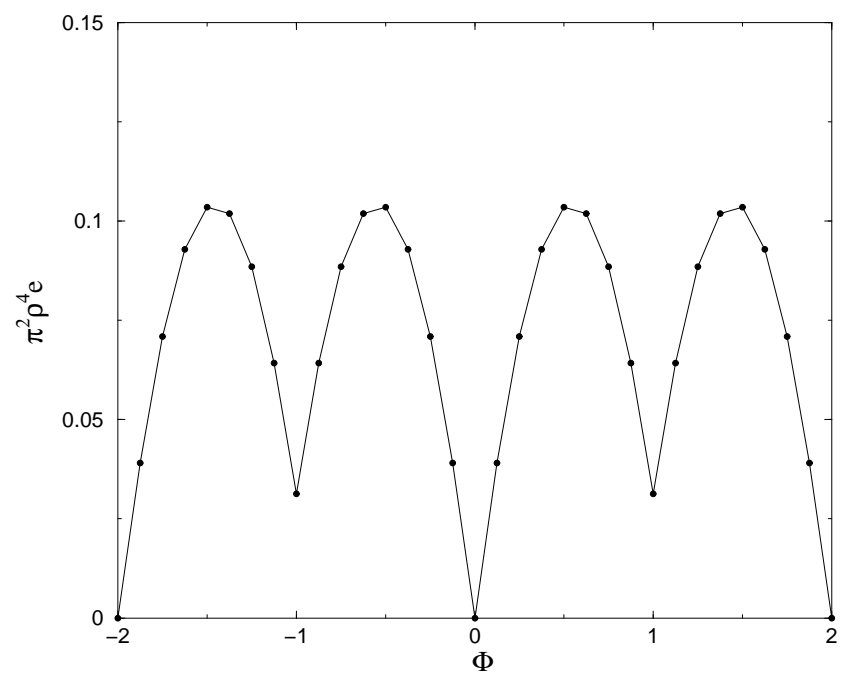

FIG. 2: Total energy density of the fluctuating gauge field and of the fermions.

Globally, the thin center vortices we consider are degenerate with the perturbative vacuum if one takes into account only gluonic fluctuations, cf. Fig. 1] This is natural, since these infinitely thin, odd integer $\Phi$ configurations correspond to pure gauge transformations in pure Yang-Mills theory - they are just as invisible to gluonic fluctuations as Dirac strings (even integer $\Phi$ ), since gluons are defined in the adjoint representation of the gauge group. This degeneracy is lifted when quark fluctuations are included. The energy density of a center vortex is raised above the energy density of the perturbative vacuum through the influence of the fermions (but it continues to represent a local minimum as a function of magnetic flux $\Phi)$. Only Dirac strings are then still degenerate with the perturbative vacuum. In a realistic vortex model of the QCD vacuum, energy densities of the type considered here are but one among a whole set of factors influencing vortex dynamics. For example, realistic vortices are not infinitely thin, but are smeared out to a finite transverse thickness, a case which has recently also been discussed 12, 13]. As shown there, a finite thickness can actually lead to a lowering of the effective energy density below the one of the perturbative vacuum. On the other hand, also vortex interactions have to be considered, cf. 22] for an investigation on the lattice. In the phenomenologically successful random vortex world-surface model 4, 6, 11], the energy associated with vortex curvature was found to play an important role - this contribution has not been investigated from first principles. In that model, 
it is ultimately the entropy inherent in the random vortex world-surface ensemble which overcomes any energy penalties associated with the formation of center vortices, and thus leads to the formation of the percolating vortex vacuum and a comprehensive description of the principal nonperturbative phenomena characterizing the strong interaction.

\section{Acknowledgments}

We thank P.A.G. Pisani for helpful discussions, especially about the proof given in Appendix A

\section{APPENDIX A: CLOSURE OF $\hat{C}$}

In this Appendix we discuss the closure of the operator $\hat{C}$, cf. (21). We prove that functions $f \in D(\overline{\hat{C}})$ are absolute continuous close to the origin and fulfill $\lim _{x \rightarrow 0} x^{-\delta} f(x)=0$, where $\delta=|m-\lambda(a) \Phi|$. To obtain $D(\overline{\hat{C}})$, we add to the domain of $\hat{C}, D(\hat{C})=C_{c}^{\infty}\left(\mathbb{R}_{>0}\right)$, the limit points of Cauchy sequences in $D(\hat{C})$, whose images under $\hat{C}$ are also Cauchy sequences. We consider such a Cauchy sequence $f_{k}$ and denote $f_{k l}=f_{k}-f_{l}$. We obtain

$$
\int_{0}^{\infty} \mathrm{d} x\left(x{f_{k l}^{\prime}}^{2}+\frac{\delta}{x}{f_{k l}}^{2}\right)=\int_{0}^{\infty} x \mathrm{~d} x f_{k l} \hat{C} f_{k l}<\epsilon, \text { for } k, l>N_{\epsilon} .
$$

Therefore, $x^{-1 / 2} f_{k}$ and $x^{1 / 2} f_{k}^{\prime}$ are Cauchy sequences in $L^{2}\left(\mathbb{R}_{>0}, \mathrm{~d} x\right)$. Observe that also $x^{1 / 2} f_{k}$ and $x^{1 / 2} \hat{C} f_{k}$ are Cauchy sequences in $L^{2}\left(\mathbb{R}_{>0}, \mathrm{~d} x\right)$.

Next we prove that if $x^{n} f_{k}^{\prime}$ and $x^{n-1} f_{k}$ are Cauchy sequences $(n \in \mathbb{R})$ in $L^{2}\left(\mathbb{R}_{>0}, \mathrm{~d} x\right)$, then also $x^{n / 2-1 / 4} f_{k}^{\prime}$ and $x^{n / 2-5 / 4} f_{k}$ are Cauchy sequences. From that, it follows by induction that $x^{-1 / 2+\epsilon} f_{k}^{\prime}$ and $x^{-3 / 2+\epsilon} f_{k}$ are Cauchy sequences for any $0<\epsilon \ll 1$. We consider $\int_{0}^{\infty} \mathrm{d} x x^{n} f_{k l}^{\prime} \sqrt{x} \hat{C} f_{k l}<\xi$ for $k, l>N_{\xi}$ and obtain by partial integration

$$
\int_{0}^{\infty} \mathrm{d} x\left(\left(\frac{n}{2}-\frac{3}{4}\right) x^{n-1 / 2} f_{k l}^{\prime 2}-\frac{\delta}{2}\left(n-\frac{3}{2}\right) x^{n-5 / 2} f_{k l}^{2}\right)<\xi .
$$

We obtain another relation from $\int_{0}^{\infty} \mathrm{d} x x^{n-1} f_{k l} \sqrt{x} \hat{C} f_{k l}<\xi$, for $k, l>N_{\xi}$ :

$$
\int_{0}^{\infty} \mathrm{d} x\left(x^{n-1 / 2}{f_{k l}^{\prime 2}}^{2}+\left(\delta-\frac{(n-3 / 2)^{2}}{2}\right) x^{n-5 / 2}{f_{k l}}^{2}\right)<\xi .
$$


For $\delta \notin \mathbb{Q}, n \in \mathbb{Q}$ and vice versa, which can be achieved for any $0 \leq \delta<1$ by adding an infinitesimal number to $n$, these two relations are linearly independent, as one can check by calculating the determinant. In these cases $x^{n / 2-5 / 4} f_{k l}$ and $x^{n / 2-1 / 4} f_{k l}^{\prime}$ are Cauchy sequences in $L^{2}\left(\mathbb{R}_{>0}, \mathrm{~d} x\right)$.

In the next step, we prove that the functions in $D(\overline{\hat{C}})$ are absolute continuous close to the origin. We consider an interval $(0, a)$ and denote the limit of $\sqrt{x} f_{k}$ by $f$, the limit of $\sqrt{x} \hat{C} f_{k}$ by $f_{\hat{C}}$, the limit of $x^{-1 / 2+\epsilon} f_{k}^{\prime}$ by $g$ and the limit of $x^{-3 / 2+\epsilon} f_{k}$ by $\tilde{g}$ in $L^{2}\left(\mathbb{R}_{>0}, \mathrm{~d} x\right)$. We get

$$
\int_{0}^{a} \mathrm{~d} x\left|f_{k}-x^{3 / 2-\epsilon} \tilde{g}\right|^{2} \leq a^{3-2 \epsilon} \int_{0}^{a} \mathrm{~d} x\left|x^{-3 / 2+\epsilon} f_{k}-\tilde{g}\right|^{2}<\xi
$$

that is

$$
f_{k} \rightarrow x^{3 / 2-\epsilon} \tilde{g} \equiv h
$$

in $L^{2}((0, a), \mathrm{d} x)$. On the other hand, as $g \in L^{2}((0, a), \mathrm{d} x)$ and therefore the integral $\int_{0}^{x} \mathrm{~d} x^{\prime} x^{\prime 1 / 2-\epsilon} g$ is well defined for all $x \in(0, a)$, we have

$$
\left|\int_{0}^{x} \mathrm{~d} x^{\prime} x^{1 / 2-\epsilon} g-f_{k}\right|^{2} \leq a^{1-2 \epsilon} \int_{0}^{x} \mathrm{~d} x^{\prime}\left|g-x^{-1 / 2+\epsilon} f_{k}^{\prime}\right|^{2}<\xi
$$

and see that

$$
f_{k} \rightarrow \int_{0}^{x} \mathrm{~d} x^{\prime} x^{1 / 2-\epsilon} g .
$$

However, as $f_{k} \rightarrow h$ we see that $h$ is absolute continuous in $(0, a)$ and $\frac{h}{x^{1-2 \epsilon}}$ is absolute continuous in $\left(a^{\prime}, a\right)$ for $0<a^{\prime}<a$. Taking the derivative of $\frac{h}{x^{1-2 \epsilon}}$ we obtain

$$
\left(\frac{h}{x^{1-2 \epsilon}}\right)^{\prime}=g \frac{1}{x^{1 / 2-\epsilon}}-(1-2 \epsilon) \tilde{g} \frac{1}{x^{1 / 2-\epsilon}} \in L^{2}((0, a), \mathrm{d} x)
$$

as all functions on the right side are in $L^{2}((0, a), \mathrm{d} x)$. Therefore

$$
\int_{0}^{a} \mathrm{~d} x\left(\frac{h}{x^{1-2 \epsilon}}\right)^{\prime}=\frac{h(a)}{a^{1-2 \epsilon}}-\lim _{a^{\prime} \rightarrow 0} \frac{h\left(a^{\prime}\right)}{a^{\prime 1-2 \epsilon}}
$$

has to be finite and we arrive at

$$
\lim _{a^{\prime} \rightarrow 0} h\left(a^{\prime}\right) a^{\prime-1+2 \epsilon}=\text { const }<\infty
$$


for any $0<\epsilon \ll 1$, i.e.

$$
x^{-\delta} h(x) \rightarrow 0 \text { for } x \rightarrow 0
$$

for all $0 \leq \delta<1$

[1] G. 't Hooft, Nucl. Phys. B138 (1978) 1;

H.B. Nielsen and P. Olesen, Nucl. Phys. B160 (1979) 380;

J. Ambjørn and P. Olesen, Nucl. Phys. B170 (1980) 60;

J. Ambjørn and P. Olesen, Nucl. Phys. B170 (1980) 265.

[2] L. Del Debbio, M. Faber, J. Greensite and Š. Olejnik, Phys. Rev. D 55 (1997) 2298;

L. Del Debbio, M. Faber, J. Giedt, J. Greensite and Š. Olejnik, Phys. Rev. D 58 (1998) 094501.

[3] K. Langfeld, O. Tennert, M. Engelhardt and H. Reinhardt, Phys. Lett. B452 (1999) 301;

M. Engelhardt, K. Langfeld, H. Reinhardt and O. Tennert, Phys. Rev. D 61 (2000) 054504.

[4] M. Engelhardt and H. Reinhardt, Nucl. Phys. B585 (2000) 591.

[5] M. Engelhardt and H. Reinhardt, Nucl. Phys. B567 (2000) 249.

[6] M. Engelhardt, Nucl. Phys. B585 (2000) 614.

[7] R. Bertle, M. Engelhardt and M. Faber, Phys. Rev. D 64 (2001) 074504

[8] H. Reinhardt, Nucl. Phys. B628 (2002) 133.

[9] J. M. Cornwall, Phys. Rev. D 65 (2002) 085045

[10] P. de Forcrand and M. D’Elia, Phys. Rev. Lett. 82 (1999) 4582;

C. Alexandrou, M. D’Elia and P. de Forcrand, Nucl. Phys. Proc. Suppl. 83 (2000) 437.

[11] M. Engelhardt, Nucl. Phys. B638 (2002) 81.

[12] D. Diakonov and M. Maul, Phys. Rev. D 66 (2002) 096004.

[13] M. Bordag, hep-th/0211080.

[14] D. Diakonov, Mod. Phys. Lett. A 14 (1999) 1725.

[15] M. Reed and B. Simon, Methods of Modern Mathematical Physics, Vols. I-II, Academic Press, 1980.

[16] I.S. Gradshteyn and I.M. Ryzhik, Table of Integrals, Series and Products, Academic Press, 1980. 
[17] P. de S. Gerbert, Phys. Rev. D 40 (1989) 1346.

[18] H. Falomir and P.A.G. Pisani, J. Phys. A: Math. Gen. 34 (2001) 4143.

[19] C. Manuel and R. Tarrach, Phys. Lett. B301 (1993) 72.

[20] Y.A. Sitenko, Phys. Lett. B387 (1996) 334.

[21] C.G. Beneventano, M. De Francia and E.M. Santangelo, Int. J. Mod. Phys. A 14 (1999) 4749.

[22] M. Engelhardt, K. Langfeld, H. Reinhardt and O. Tennert, Phys. Lett. B431 (1998) 141. 